\title{
Synthesis of 2D transition metal dichalcogenides by chemical vapor deposition with controlled layer number and morphology
}

\author{
Jiawen You, Md Delowar Hossain and Zhengtang Luo*
}

\begin{abstract}
Two-dimensional (2D) transition metal dichalcogenides (TMDs) have stimulated the modern technology due to their unique and tunable electronic, optical, and chemical properties. Therefore, it is very important to study the control parameters for material preparation to achieve high quality thin films for modern electronics, as the performance of TMDs-based device largely depends on their layer number, grain size, orientation, and morphology. Among the synthesis methods, chemical vapor deposition (CVD) is an excellent technique, vastly used to grow controlled layer of 2D materials in recent years. In this review, we discuss the different growth routes and mechanisms to synthesize high quality large size TMDs using CVD method. We highlight the recent advances in the controlled growth of mono- and few-layer TMDs materials by varying different growth parameters. Finally, different strategies to control the grain size, boundaries, orientation, morphology and their application for various field of are also thoroughly discussed.
\end{abstract}

Keywords: Transition metal dichalcogenides (TMDs), 2D materials, Growth mechanisms, Chemical vapor deposition, Morphology

\section{Introduction}

In the last decade, after tremendous success of the first two-dimensional (2D) material, i.e. graphene, transition metal dichalcogenides (TMDs) have attracted significant research attention due to their extraordinary properties. The TMDs represent a large family of layered materials with a generalize single layer formula as $\mathrm{MX}_{2}$, where $\mathrm{M}$ represents a transition metal from group IVB to group VIII (layered structures are usually found in group IVB to VII B, like Ti, V, Nb, Mo, W, Re), and X represents the chalcogen (S, Se, and Te) [1]. Compared with graphene, bulk TMDs show much wide range of physical properties, starting from insulators (e.g., $\mathrm{HfS}_{2}$ ) [2], semiconductors (e.g., $\mathrm{MoS}_{2}, \mathrm{WS}_{2}$ ) [3, 4], and semi metallics (e.g., $\mathrm{WTe}_{2}$ and $\mathrm{TiSe}_{2}$ ) $[5,6]$ to metallics (e.g., $\mathrm{NbS}_{2}, \mathrm{VSe}_{2}$ ) [7, 8]. Apart from this, a few of them (e.g., $\mathrm{NbSe}_{2}, \mathrm{TaS}_{2}$ ) shows unusual interesting properties like superconductivity,

\footnotetext{
*Correspondence: keztluo@ust.hk

Department of Chemical and Biological Engineering, Hong Kong

University of Science and Technology, Clear Water Bay, Kowloon, Hong Kong
}

charge density wave (CDW, periodic crystal lattice distortion) and Mott transition (transition from metal to non-metal) [9-11]. However, mono- or few-layer TMDs exhibits other additional exciting electronics characteristics alone with abovementioned properties. As for example, monolayer $\mathrm{MoS}_{2}$ shows strong photoluminescence (PL) over bulk due to quantum confinements, confirmed by the change of bandgap from indirect to direct for bulk to monolayer $\mathrm{MoS}_{2}$ [12]. Further research also indicates that the CDW transition temperature of $\mathrm{NbSe}_{2}$ increases on reducing the layer number due to the enhanced electron-phonon interactions in 2D materials [13]. Due to numerous unique properties of thin layer TMDs materials, it has been used to fabricate new generation electronic [14-16] and optoelectronic devices [17-20] such as transistors, photodetector, photodiode, photovoltaic devices, etc. Therefore, researchers have devoted considerable efforts to synthesis high-quality and large-size TMDs thin layers, which plays a significant role in fundamental research and application exploration. Several techniques have been already introduced to synthesize atomically thin layer of high quality TMDs material such 
as mechanical exfoliation method [21, 22], liquid exfoliation method [23-25], chemical vapor deposition (CVD) method [26-29], wet chemical method [30, 31], etc. Among all synthesis techniques, the chemical vapor deposition (CVD) is promising for synthesis of high-quality TMDs layers with controllable layer number and domain size, as well as excellent properties, due to their simplicity and compatibility with industry standards.

In this review, we summarized some of the key and control factors affecting the growth of TMDs via CVD method. Here, CVD is used as a general term which covers the vapor transport and deposition methods. The growth mechanisms and synthesis approaches of monoor few-layer TMDs are also be introduced. Moreover, we will thoroughly discuss the controllable growth of TMDs based on grain size/boundary, orientation, and morphology.

\section{Growth routes and mechanisms}

Different strategies have been applied in the synthesis technique to obtain high quality large scale mono- and few-layer TMDs to explore their promising properties in various field of applications. Typically, all the synthesis methods have been classified into either "top-down" or "bottom-up" approaches [32]. Generally, exfoliation methods are followed "top-down" technique while CVD is based on "bottom-up" methods [33-35]. In a typical CVD process, precursors are reacted/or decomposed and deposited as mono- or few-layer film on the exposed substrate at relative high temperature. Many fundamental researches have been done to produce ultrathin TMDs materials with high crystal quality, scalable size, tunable thickness, and excellent electronic properties [26, 36, 37]. Compared with chemical vapor transport (CVT) method, which is commonly used to synthesize bulk single crystal materials, the typical CVD method we discussed in this review is for synthesizing mono- or few-layer TMDs. The reaction process is much quicker than CVT, and no subsequent mechanical exfoliation is required to obtain few layer structure for following study and application.

\subsection{Synthesis routes for TMDs layers}

There are four common routes of TMDs growth by CVD approaches that has been reported up to date. (1) The first route is thermal decomposition of precursors [using compounds like $\left(\mathrm{NH}_{4}\right)_{2} \mathrm{MoS}_{4}$, consists of both metal and chalcogen elements] under reductive and inert environment, while the presence of $\mathrm{H}_{2}$ at low temperature avoids oxidation and converts $\left(\mathrm{NH}_{4}\right)_{2} \mathrm{MoS}_{4}$ precursor directly into $\mathrm{MoS}_{2}$ [38]. Normally, a two-step thermolysis process was used to obtain high-quality thin film, as shown in Fig. 1a. After dip-coating of $\left(\mathrm{NH}_{4}\right)_{2} \mathrm{MoS}_{4}$ on substrates, $\mathrm{Ar} / \mathrm{H}_{2}$ mix flow were introduced and kept at relative low temperature and low pressures $\left(500{ }^{\circ} \mathrm{C}, 1\right.$ Torr) for an hour to remove the residual solvent, $\mathrm{NH}_{3}, \mathrm{H}_{2} \mathrm{~S}$ and other byproducts; then at second step of annealing, high temperature $\left(1000{ }^{\circ} \mathrm{C}\right)$ and additional sulfur were applied to improve the crystallinity and electrical performance. The extra sulfur helped to remove excess oxygen and mitigate sulfur deficit caused by organic solvent evaporation [39]. Based on this, wafer scale $\mathrm{MoS}_{2}$ thin films were synthesized by incorporating chelating agent like ethylenediaminetetraacetic acid (EDTA) with $\left(\mathrm{NH}_{4}\right)_{2} \mathrm{MoS}_{4}$ in DMSO solvent as a spin-coating solution, followed by heat treatment in furnace at $500-800{ }^{\circ} \mathrm{C}$ and Ar atmosphere [40]. Similarly, a series of $\mathrm{rGO} / \mathrm{XS}_{2}$ heterostructures $(\mathrm{X}=\mathrm{W}, \mathrm{Mo}$, or $\mathrm{W}$ and Mo alloy) were synthesized by dispersing $\left(\mathrm{NH}_{4}\right)_{2} \mathrm{WS}_{4}$ or $\left(\mathrm{NH}_{4}\right)_{2} \mathrm{MoS}_{4}$ into a GO solution followed by thermal treatment at low temperature [41]. (2) The second route is by sulfurization/selenization/tellurization of pre-deposited metal or metal oxide films on suitable substrate. Some techniques, such as e-beam evaporation [28], spin coating [42], atomic layer deposition (ALD) [43], and thermal evaporation [44] have been used to deposit metal or metal-containing precursors. In a typical growth, rhomboidal $\mathrm{MoO}_{2}$ microplates nucleated and grew on substrate through thermally evaporation, and then reduced by sulfur vapor at 650 $850{ }^{\circ} \mathrm{C}$, then $\mathrm{MoO}_{2}$ were sulfurized to $\mathrm{MoS}_{2}$ layer-bylayer at higher temperature (Fig. 1b). Because the $\mathrm{MoO}_{2}$ microplates were more crystalline and much larger than $\mathrm{MoO}_{2}$ nanoparticles, the layer-by-layer sulfurization process was much slower, which allowed to control the layer numbers by changing the high-temperature annealing time. The obtained high-quality micrometer size $\mathrm{MoS}_{2}$ rhomboid flakes show comparable performance with mechanically exfoliated products after being used into back-gated field effect transistors (FETs) device [45]. A continuous uniform $\mathrm{WS}_{2}$ films were synthesized by decorating silicon wafer through silanization reaction to improve the dispersion of precursors $\left(\mathrm{WO}_{3} \cdot \mathrm{xH}_{2} \mathrm{O}\right)$, then a continuous $\mathrm{WO}_{3}$ formed after evaporating the solvent [46]. (3) The third route includes a simple physical vapor transport method to grow high quality monolayer or fewlayers TMDs. For example, the $\mathrm{MoS}_{2}$ powder are involved as precursor which was kept at higher temperature zone $\left(\sim 900^{\circ} \mathrm{C}\right)$ and finally high quality $\mathrm{MoS}_{2}$ was deposited on insulating substrate (placed at cooler zone, $\sim 650^{\circ} \mathrm{C}$ ) via vapor-solid growth mechanism under Ar gas (Fig. 1c) [47]. (4) The fourth route to synthesize TMDs materials by CVD method involves vapor phase reaction of two precursor e.g., transition metal oxides/halides and chalcogen precursors. This kind of CVD process is classified into atmospheric pressure CVD (APCVD), modified metal-organic CVD (MOCVD) and low-pressure CVD (LPCVD) (Fig. 1d (i)) etc., and these are the most 
a

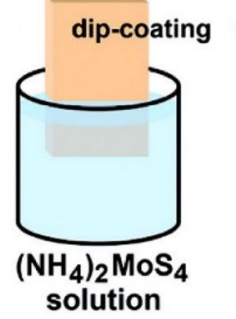

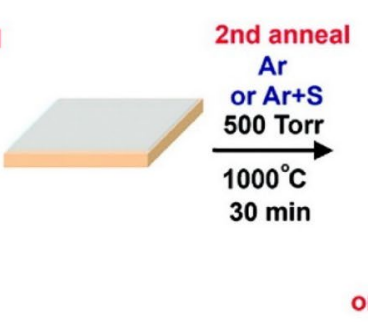
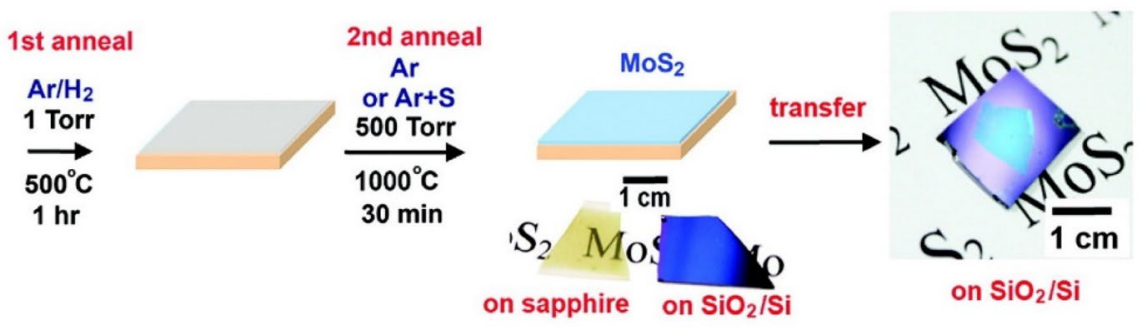

b

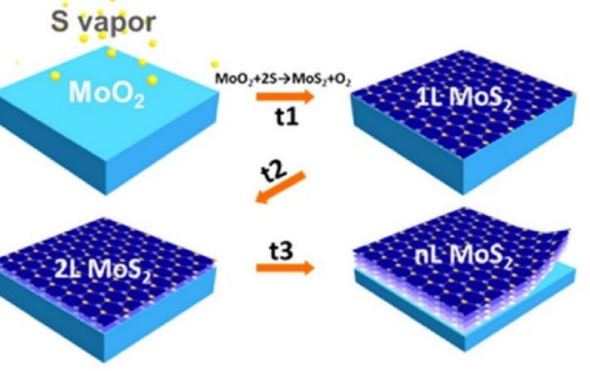

c
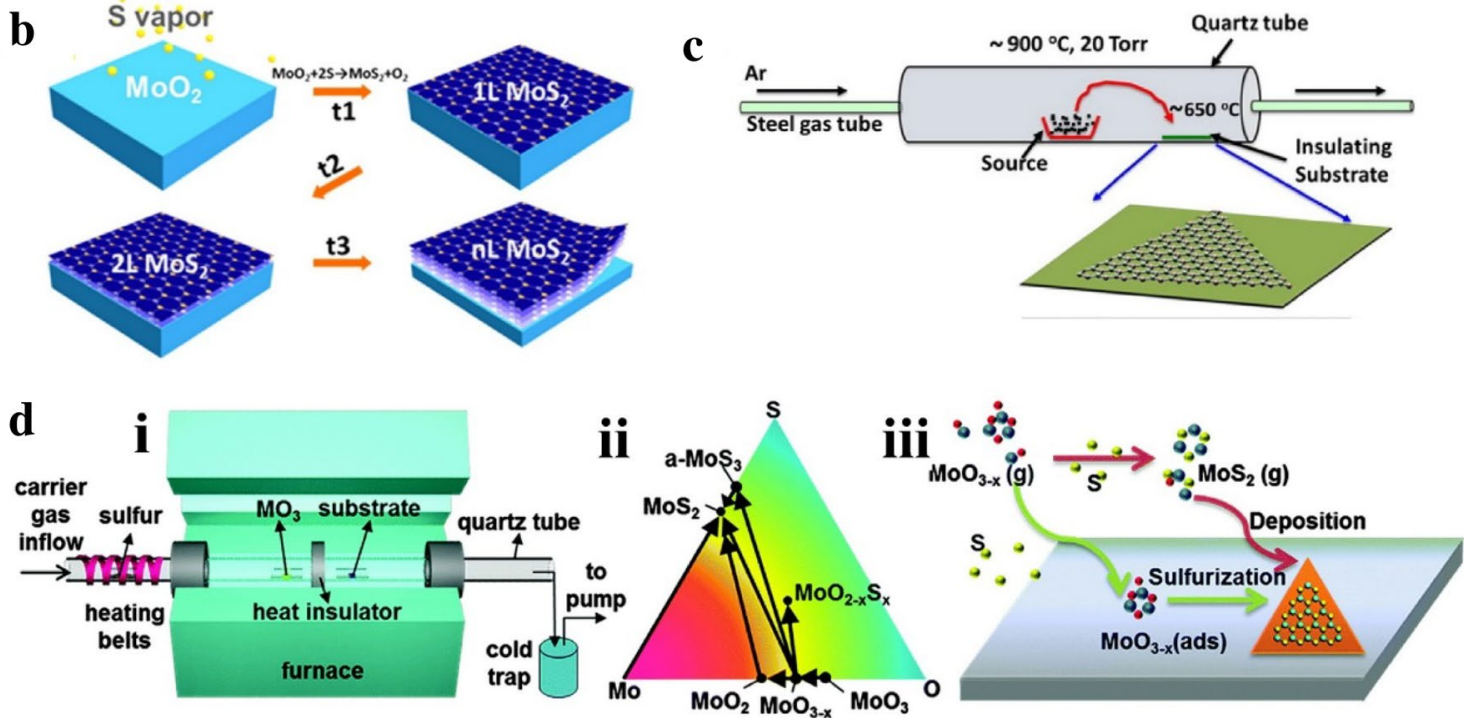

Fig. 14 types of growth routes for TMDs. a Two-step thermolysis process synthesis from $\left(\mathrm{NH}_{4}\right)_{2} \mathrm{MoS}_{4}$ precursor on $\mathrm{SiO}_{2} / \mathrm{Si}$ or sapphire substrates followed by the two-step annealing process on insulating substrates and finally transferred onto another substrate [39], Copyright 2012, American Chemical Society. $\mathbf{b}$ Layer by layer deposition of $\mathrm{MoS}_{2}$ from $\mathrm{MoO}_{3}$ by surface sulfurization and transferred to another substrate one by one [45], Copyright 2013, American Chemical Society. c Simple vapor transport method showing MoS $_{2}$ films were deposited on insulating substrate at high temperature from $\mathrm{MoS}_{2}$ powder precursor under inert atmosphere [47], Copyright 2013, American Chemical Society. d (i) LPCVD system setup for TMDs, (ii) Mo-O-S ternary phase diagram illustrating the pathways for the CVD growth of $\mathrm{MoS}_{2}$ from $\mathrm{MoO}_{3}$ precursors, and (iii) Possible growth routes of $\mathrm{MoS}_{2}$ by the reaction of $\mathrm{MoO}_{3-x}$ and S [36]. Copyright 2015, Royal Society of Chemistry

common techniques to synthesize TMDs layers. For CVD growth of $\mathrm{MoS}_{2}$ from $\mathrm{MoO}_{3}$ and sulfur powder, the Mo-O-S ternary phase diagram (Fig. 1d (ii)) and reaction pathways have been summarized by recent references [36]. It has been considered that $\mathrm{MoO}_{3}$ reacts with sulfur and produces $\mathrm{MoS}_{2}$ (Fig. 1d (iii)) according to the following two-step reaction,

$$
\begin{aligned}
& 2 \mathrm{MoO}_{3}+\mathrm{xS} \rightarrow \mathrm{MoO}_{3-\mathrm{x}}+\mathrm{xSO}_{2} \\
& 2 \mathrm{MoO}_{3-\mathrm{x}}+(7-\mathrm{x}) \mathrm{S} \rightarrow \mathrm{xMoS}_{2}+(3-\mathrm{x}) \mathrm{SO}_{2} .
\end{aligned}
$$

But, several researchers have found that $\mathrm{H}_{2}$ also plays a vital role for the growth of TMDs. It acts as an additional reducing agent with Se during the growth of $\mathrm{MoSe}_{2}$ nanosheets [48] and thin films [49] when solid Se and $\mathrm{MoO}_{3}$ are used as precursors. Similarly, it has also been proven that $\mathrm{H}_{2}$ plays an important role for thermodynamics of the selenization of $\mathrm{WO}_{3}$ following the chemical reaction [50],

$$
\mathrm{WO}_{3}+3 \mathrm{Se}+\mathrm{H}_{2} \rightarrow \mathrm{WSe}_{2}+\mathrm{H}_{2} \mathrm{O}+\mathrm{SeO}_{2}
$$

On the other hand, due to the lower melting point of metal halides over metal sources, it is more efficient to control the ratio of metal and chalcogen. High quality single crystalline $3 \mathrm{R}-\mathrm{MoTe}_{2}$ flakes were synthesized by CVD method by using $\mathrm{MoCl}_{5}$ as Mo precursor [51]. Very recently, molten-salt-assisted CVD method has reported for synthesizing a series of (2D) transition-metal chalcogenides (TMCs), especially for some high melting points, low vapor pressure metal and metal oxides precursors. The addition of molten salts reacted with metal precursors, and form intermediate oxychlorides which has lower melting points, thus the mass flux and reaction rate 
successfully is increased. This method has demonstrated to be able to synthesize 47 kinds of transition-metal chalcogenides (TMCs), including 32 binary compounds, 13 alloys, and 2 hetero-structures [52]. Similarly, some graphene-like molecules, such as reduced graphene oxide (rGO), perylene-3,4,9,10 tetracarboxylic acid tetra-potassium salt (PTAS) and perylene-3,4,9,10-tetracarboxylic dianhydride (PTCDA) can be used as growth seeds and promoted the $\mathrm{MoS}_{2}$ layer growth [26]. Further research have also investigated how the seeding promoters and seed concentration influence the growth of $\mathrm{MoS}_{2}$ monolayer [53]. It is believed that different kinds of aromatic molecules acted as a seeding promoters, deposited on various substrates and increased the surface adhesive force of $\mathrm{MoS}_{2}$ to improve the layer growth of $\mathrm{MoS}_{2}$.

\subsection{Thermodynamics and Kinetics for TMDs Growth}

Three growth modes have been identified for different kinds TMDs materials including island growth (Volmer-Weber growth mechanism), layer-by-layer growth (Frank-vander Merwe growth mechanism), and mixed growth (Stranski-Krastanov growth mechanism). It is well recognized that the integration of thermodynamics and kinetics with nucleation growth provided important guidance to understand the growth mechanism as well as TMDs layers control. Hence, in this review, we mainly discuss thermodynamics and kinetics for the growth of 2D TMDs, and several mechanistic models have been introduced based on those thermodynamic and kinetic mechanisms.

At the early stage, the thermodynamics of $\mathrm{MoS}_{2}$ deposition by reaction of $\mathrm{H}_{2} \mathrm{~S}$ with molybdenum fluoride or chloride had been thoroughly studied. The chemical equilibria for Mo-S-CI-H and Mo-S-F-H system were calculated at $1 \mathrm{kPa}$ as a function of temperature and reagent molar ratio $(\psi), \mathrm{H}_{2} \mathrm{~S} /\left(\mathrm{MoF}_{6}+\mathrm{H}_{2} \mathrm{~S}\right)$ or $\mathrm{H}_{2} \mathrm{~S} /$ $\left(\mathrm{MoCl}_{4}+\mathrm{H}_{2} \mathrm{~S}\right)$. The results indicated that the $\mathrm{MoS}_{2}$ was thermodynamically favorable to deposit in the presence of excess $\mathrm{H}_{2} \mathrm{~S}$ [54]. Recent work proposed that partial pressure of gaseous $\mathrm{MoS}_{2}\left(\mathrm{P}_{\mathrm{Mo}}\right)$ plays an important role in the control of layer number due to the thermodynamic and kinetic effects on precipitation reaction. The experimental results showed that the partial pressure must be larger than the vapor pressure $\left(\mathrm{P}_{\mathrm{Mo}}^{\mathrm{o}}\right)$, using the pressure difference provides the thermodynamic driving force for the precipitation reaction. So, by controlling $\mathrm{P}_{\mathrm{Mo}}$, the layer number can be controlled. In particular, it is found that when $\mathrm{P}_{\mathrm{Mo}}$ is between the vapor pressure of monolayer and bilayer $\left(\mathrm{P}_{\mathrm{Mo}, 1}^{\mathrm{o}}<\mathrm{P}_{\mathrm{Mo}}<\mathrm{P}_{\mathrm{Mo}, 2}^{\mathrm{o}}\right)$, exclusively monolayer were obtained. However, the larger $\mathrm{P}_{\mathrm{Mo}}$ leaded to the faster precipitation [55]. According to the DFT calculated relative energies of layer-dependence of $\mathrm{MoS}_{2}$, and flake-size dependence of mono/bilayer $\mathrm{MoS}_{2}$, it has been demonstrated that the small monolayer $\mathrm{MoS}_{2}$ flakes were thermodynamically favorable during the initial stage of nucleation; while with the increasing of lateral size, the vertical growth become preferable. The relative energies of the semi-infinite slabs with and without graphene substrate had also been calculated in the same work and found that graphene substrate can greatly increase the critical size of monolayer flake [56]. Furthermore, the size-dependent pressure-temperature-composition ( $\mathrm{P}-\mathrm{T}-\mathrm{x}$ ) phase diagrams (Fig. 2a, b) were provided to predict the $2 \mathrm{D}$ lateral size and thickness-dependent $\mathrm{MoS}_{2}$ growth window. In addition, the calculated migration energy and diffusivity of Mo and $\mathrm{S}$ indicating that the growth of $\mathrm{MoS}_{2}$ is controlled by Mo due to the low diffusivity respect to Sulfur. This integrated density functional theory (DFT) and calculation of phase diagram (CALPHAD) modeling approach provided a quantitative insight into the controlled lateral and vertical growth of 2D TMDs [56].

On the other hand, the growth rate of subsequent layer depends on the sizes of initial layer and subsequent layer. Recently, a multiscale model has been developed and found that a maximum size of layer-1, while minimum size of layer- 2 ensured vertical growth according to thermodynamic criterion. The increasing size of layer-1 decreased the growth rate of layer2 , while the size of layer- 1 reaching the maximum size, the growth of layer-2 ceased to grow. A successful vertical growth happens when the size of layer- 2 exceeds the critical size before layer-1 reaching maximum size. By changing the kinetic parameters (growth temperature and flux), it was demonstrated that the monolayer was obtained at relatively low temperature but high gas flow rate conditions (Fig. 2c, d), consistent with the trend of growth of bilayer graphene at decreased supply of adatoms $[57,58]$. In order to investigate and predict vdW epitaxy growth process to guide experimental study, a Kinetic Monte Carlo (KMC) simulation method was used to investigate the complex competitions in growth rate, morphology, homogeneous nucleation and layer number. Extrinsic parameters (including temperature, adsorption rate, chalcogen to metal ratio of the precursors), intrinsic parameters, site energy, adsorption energy and transition energy barriers, as well as substrate effects were introduced to provide a fundamental understanding about the growth mechanisms at atomic scale [59]. Although this model was set for molecular beam epitaxy (MBE) growth of TMDs, after further refinements it applied to predict CVD growth qualitatively and quantitatively. 

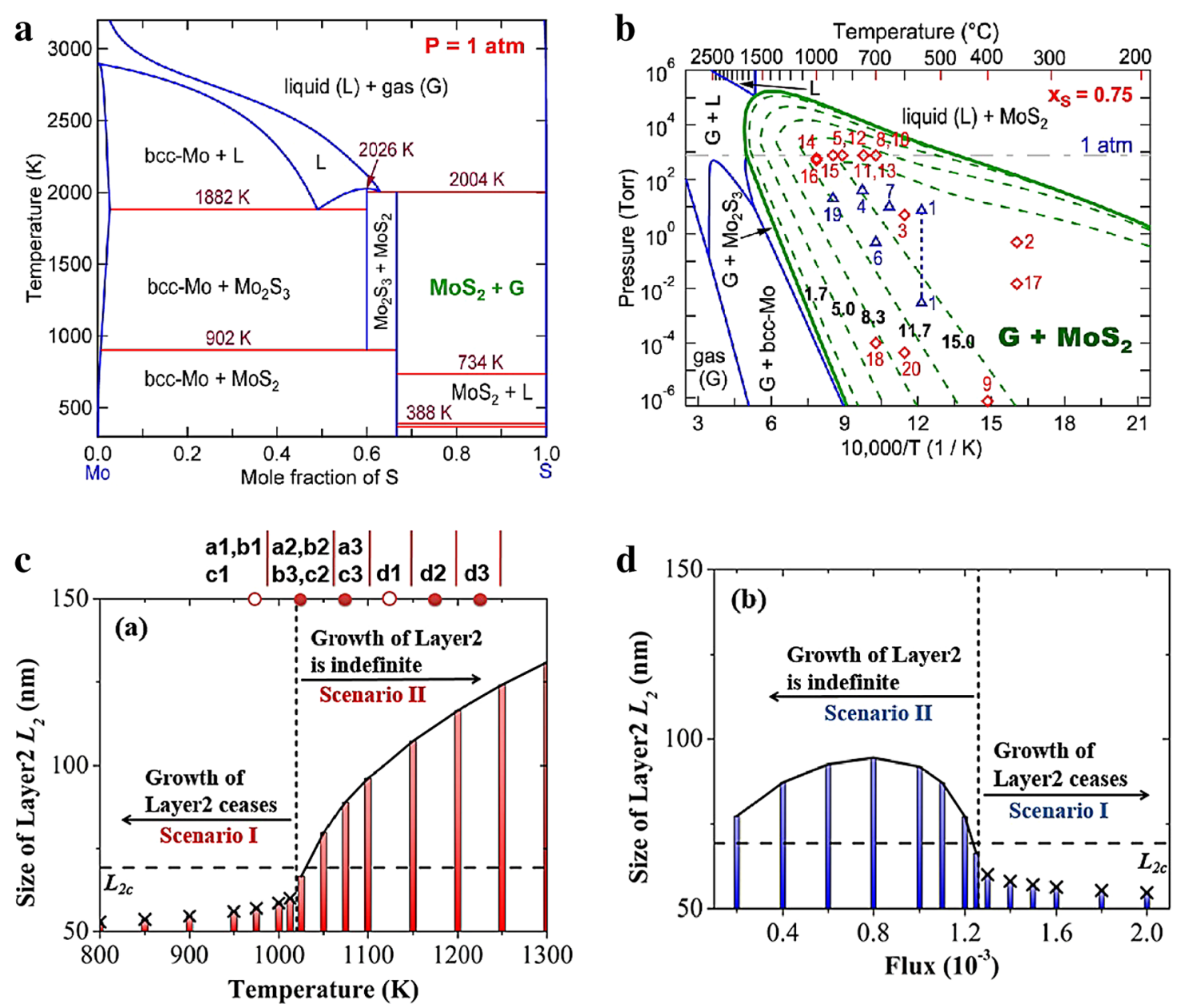

Fig. 2 Thermodynamics and kinetics during TMDs growth. $\mathbf{a}$, b CALPHAD modeled temperature-composition (T-x), and pressure-temperature (P-T) phase diagram for the Mo-S system under pressure and under the S-rich condition respectively [56]. Copyright 2016, American Chemical Society. c Effect of temperature and, d precursor flux on the growth of layer 2 for 2 s [57]. Copyright 2017, American Chemical Society

\section{Controllable growth for TMDs materials}

The potential applications in electronic and optoelectronic devices has demanded the scalable synthesis of uniform, high-quality TMDs layers. Generally, high-quality refers to high crystallinity, large domain size, defectfree, and limited grain boundaries. The quality of large scale uniform $\mathrm{MoS}_{2}$ layers largely depends on property factors such as grain size and boundaries, orientation and their morphology, as discussed below.

\subsection{Domain size and grain boundaries}

Previous report has shown that large-scale polycrystalline $\mathrm{MoS}_{2}$ film with a tunable grain size up to micrometers can be synthesized via a new CVD configuration. When the grain size increased from 20 to $600 \mathrm{~nm}$, Raman spectra showed nearly no difference in peak position and intensity, but obvious PL shift was observed due to band-gap modulation. Moreover, because of the extra scattering at grain boundaries, it was clear that the carrier mobility was impaired when grain size reduced [60]. Furthermore, recent research showed that the low angle grain boundaries of CVD-grown polycrystalline $\mathrm{MoS}_{2}$ deteriorate thermal conductivity [61]. It has widely acknowledged that the nucleation density is closely related to the grain size. The following equation shows the relationship between source and growth distance (d) with thermodynamic and kinetic growth factor-the concentration of gaseous $\mathrm{MoS}_{2}(C g)$ :

$$
C_{g}(d, t)=C_{g}(0, t) \exp \left(-\frac{d^{2}}{4 D t}\right)
$$

where $t$ is the time, and $D$ is the diffusion constant. Figure 3a summarized the surface coverage and the average domain size as a function of distance. When the distance was less than $7 \mathrm{~mm}$, high $\mathrm{Cg}$ ensured sufficient source 
a

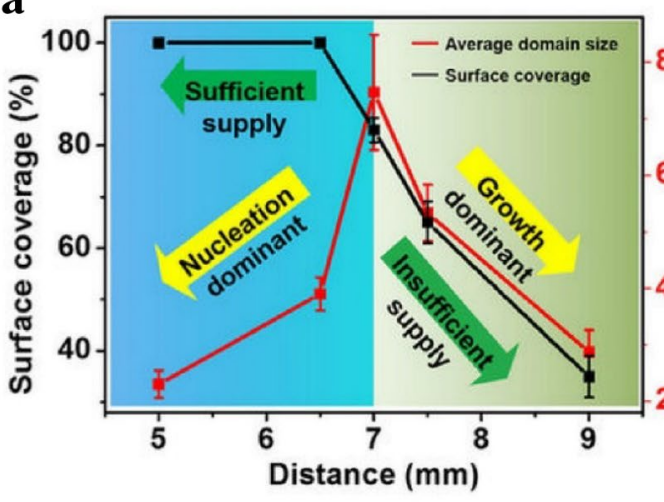

$-80$

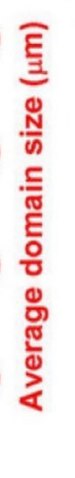

b

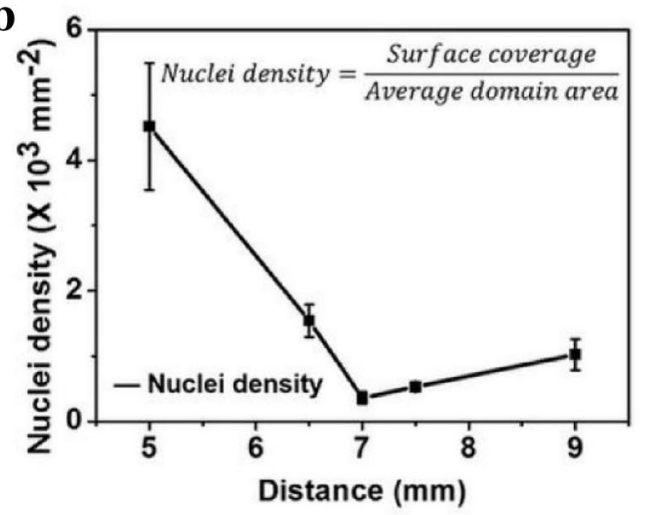

c

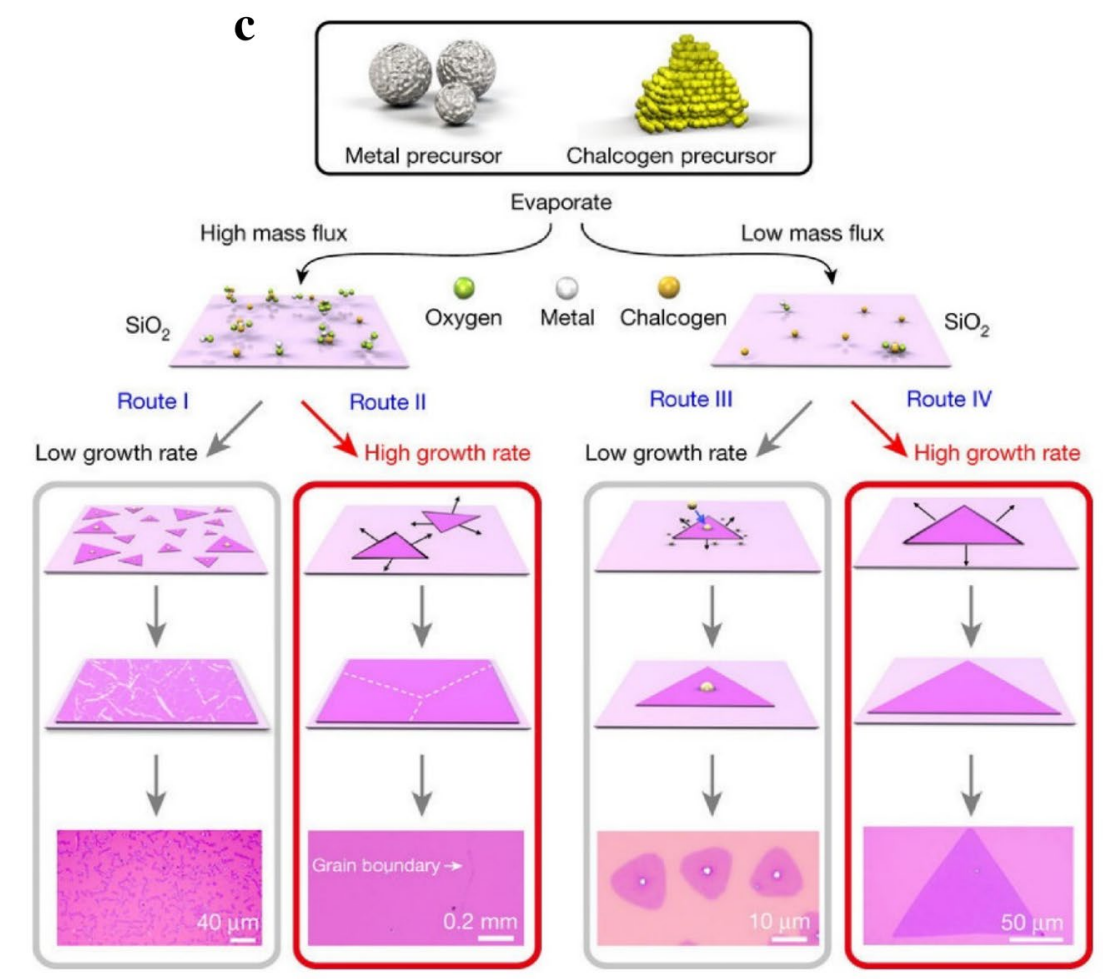

Fig. 3 Grain size and boundaries control. a Surface coverage and average domain size of $\mathrm{MoS}_{2}$ as a function of precursor/substrate distance, $\mathbf{b}$ effect of precursor/substrate distance on the nuclei density of $\mathrm{MoS}_{2}$ during growth [62], Copyright 2015, Springer Nature. c Flow diagram represents different routes for the synthesis of distinct types TMCs by the chemical vapor deposition method. Different routes indicated the nucleus formation and their growth mechanism as a function of mass flux of precursor and growth rate [52]. Copyright 2018, Springer Nature

supply and high nuclei density results many small $\mathrm{MoS}_{2}$ domains adjacent, coalescent, and overlap at boundary with high surface coverage. While the largest singlecrystal domain size $(>300 \mu \mathrm{m})$ was obtained at sufficient source supply but suppressed nuclei density condition $(d=7 \mathrm{~mm})$ (Fig. 3b). However, because of the low $C g$ at higher distance $(d>7 \mathrm{~mm})$, both average domain size and surface coverage decreased due to the insufficient source supply [62].
Depending on various mass flux and growth rate, the growth of 2D TMCs is divided into four routes (Fig. 3c). The formation of nucleus and the growth of domains depends on mass flux, especially metal precursor, while the grain size is determined by growth rate. High mass flux of metal precursor but low growth rate tends to form polycrystalline film with small grains and lots of grain boundaries (Route I), while high growth rate can produce smoother monolayer film with large grain size 
and limited grain boundaries (Route II). However, at low mass flux, it prefers to form single-crystalline with small domains at low growth rate (Route III), large monolayer single-crystal at high growth rate (Route IV) [52]. In order to decrease the nucleation density, without changing the amounts of precursors or growth parameters, some additional attempts have been successfully made. For example, $\mathrm{NiO}$ foam was used to grow centimeter scale continuous monolayer $\mathrm{MoS}_{2}$ by trapping chemical precursors [63]. Metal precursors $\left(\mathrm{MoO}_{3}\right)$ reacted with $\mathrm{NiO}$ and form a Ni-Mo complex, thus the precursor concentration as well as nucleation density decreased. Recently, "liquid-state" substrates show a new possibility to grow large grain size TMDs materials. Because of the low nucleation density together with low migration coefficient, large $\mathrm{MoSe}_{2}$ and $\mathrm{MoS}_{2}$ monolayer single-crystals up to $\sim 2.5 \mathrm{~mm}$ successfully deposited on the low-defect and homogeneous molten glass surface [64]. Similarly, another report has further demonstrated that the uniform distribution of $\mathrm{Na}$ in soda-lime glass will promote the growth rate of monolayer $\mathrm{MoS}_{2}$. A face-to-face metal precursor supply route was designed to synthesize 6-inch uniform monolayer $\mathrm{MoS}_{2}$ with grain edge length larger than $400 \mu \mathrm{m}$. The DFT calculations proved that during the $\mathrm{MoS}_{2}$ growth, the energy barrier was reduced with $\mathrm{Na}$ adsorption [65]. On the other way, due to the different facet-dependent binding energy between $\mathrm{Au}$ and $\mathrm{MoS}_{2}$, it is found that at relative high growth temperatures, large single-crystal domains prefer to grow on $\mathrm{Au}(100)$ and $\mathrm{Au}$ (110) than on Au (111) facets [66].

The grain boundary is another important factor that significantly affects the quality of layered TMDs, and therefore it is essential to understand the grain boundary formation mechanism, as well as the electric and optical performance at boundary. Two possible modes are observed for $\mathrm{MoS}_{2}$ boundary formation during vapor phase growth process. The traditional grain boundaries growth involves formation of chemical bonds between two single layer grains, where in-plane growth is stopped but the boundary site contributes to the nucleation of second layer. While the other mode of boundary formation involves no chemical bonds, with two grains overlap and continue to grow on top of each other [67]. At the same time, the optical and electronic properties of faceted tilt and mirror twin grain boundaries in polycrystalline $\mathrm{MoS}_{2}$ has been investigated, observing strong enhancement of PL and slight decrease of electrical conductivity at faceted tilt boundaries, with the opposite at the mirror twin boundaries [68]. This has been further demonstrated that the PL mapping can be used to identify grain boundaries in $\mathrm{MoS}_{2}$ quickly due to the thermal mismatch induced non-uniform tensile strain [69]. Meanwhile, it is also found that $60^{\circ}$ grain boundaries, either point sharing or edge sharing, both consists of distinct fourfold ring chains and exhibit metallic behavior. Further work has provided insight into various smallangle $\left(18.5^{\circ}, 17.5^{\circ}\right)$ grain boundaries possessing distinct kinds of dislocation core structures. The interaction of grain boundaries with point defects showed the possibility to control the precise grain boundary [70].

\subsection{Orientation}

As mentioned above, the grain boundary formed from different misorientation of adjacent grains, which leads to distinct electronic structures across the boundary [71]. In most cases, the distorted grain boundaries with high misorientation angles and defects lowered the quality of TMDs as electronic devices. Therefore, like grain-size controlled strategy, control the grains orientation as well as highly aligned growth of 2D TMDs are also important to promote the formation of large-size uniform layers. Two mechanisms have been developed to explain the dominant $60^{\circ}$ misorientation. In the first scenario, the small $2 \mathrm{D}$ islands were pre-aligned at $60^{\circ}$; while at the second scenario, the random orientations of islands were drawn by capillary forces into $60^{\circ}$ [72].

Recently, many aligned growths have been successfully achieved on single crystal substrates such as c-plane (0010) facet sapphire, mica, or GaN [73], which guided about TMDs growth orientation by step-edges at relative high temperature, or through van der Waals (vdW) interaction due to the lattice match between substrate and TMDs [74]. Moreover, it has been demonstrated that the small $\mathrm{MoS}_{2}$ seeds easily rotated to favorable orientations $\left(0^{\circ}\right.$ or $\left.60^{\circ}\right)$, due to the low potential energy between $\mathrm{MoS}_{2}$ seed and c-plane sapphire substrate. While the ratio of precursors $\left(\mathrm{S} / \mathrm{MoO}_{3}\right)$ plays a key role to form a rotatable seed at initial nucleation step (Fig. 4a) [75]. Similarly, mica substrate was used for such synthesis, shown in Fig. 4b, c and it is reported that the nearly lattice-matching property result at a relative lower growth temperature $\left(\sim 530{ }^{\circ} \mathrm{C}\right)$, and the triangle $\mathrm{MoS}_{2}$ seeds were primarily aligned along two opposite orientations $[62,76]$. Further work revealed that high concentration of hydrogen helped to form an active Al-rich sapphire surface, enhancing the interaction between $\mathrm{WS}_{2}$ and sapphire, thus formed well oriented triangle and effective stitch of merged grains [77]. Apart from single crystal substrates, the underlying high-symmetry materials, such as graphene or hexagonal boron nitride (h-BN) also used to align the triangle domains at $60^{\circ}$. Recent literatures have shown that $\mathrm{WSe}_{2}$ triangles were orientated as similar patterns on graphene, results epitaxial growth of monolayer $\mathrm{WSe}_{2}$ (Fig. 4d). On the other hand, selected area electron diffraction (SAED) results showed misorientation of $\mathrm{MoS}_{2}$ on graphene producing non-epitaxial 

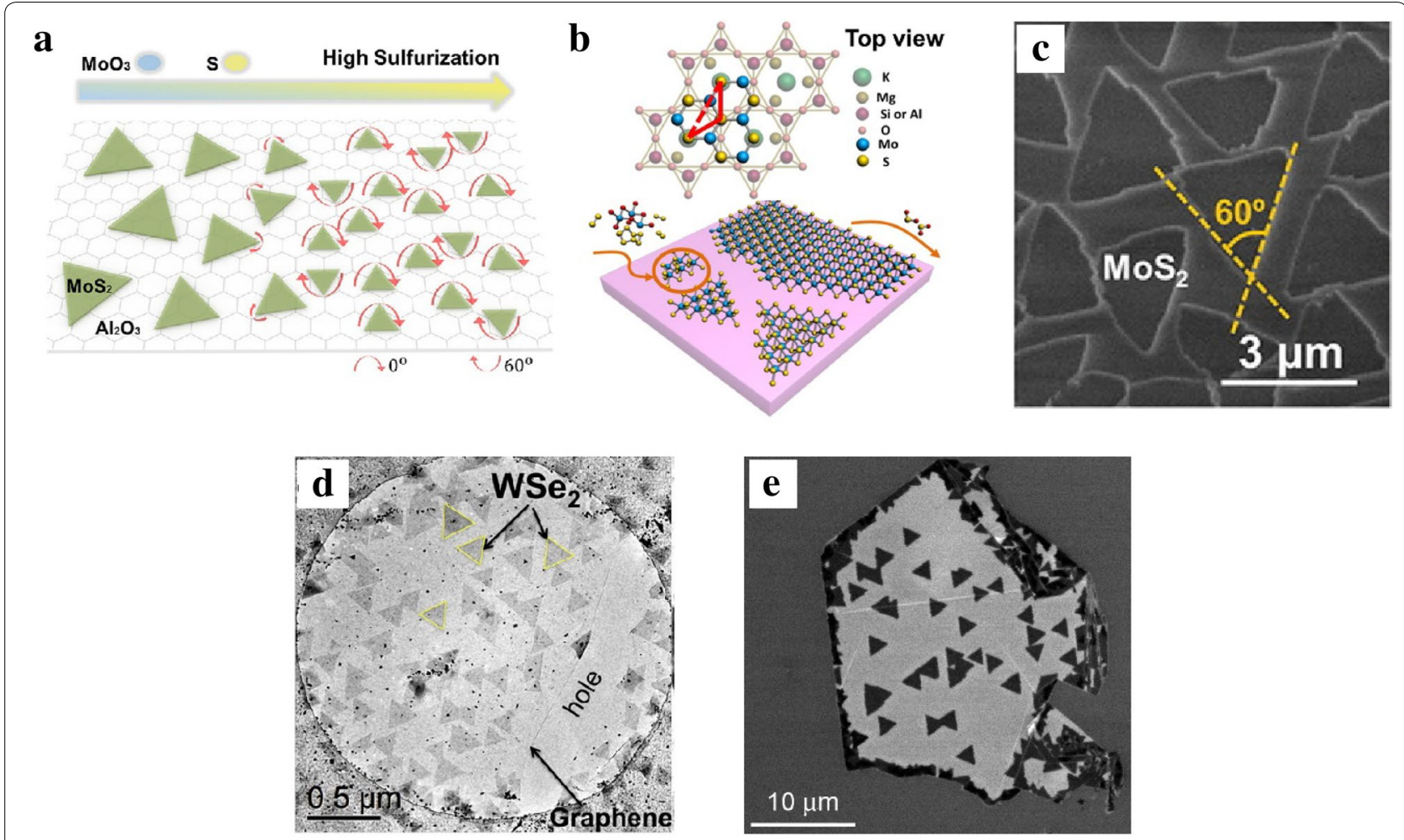

Fig. 4 Orientation control of TMDs materials. a Rotation of $\mathrm{MoS}_{2}$ seeds as a function of precursor ratio $\left(\mathrm{S} / \mathrm{MoO}_{3}\right)$ during initial nucleation steps [75]. Copyright 2017, American Chemical Society. b Epitaxial growth of $\mathrm{MoS}_{2}$ on mica surface. c SEM image of MoS 2 on mica [76]. Copyright 2013, American Chemical Society. $\mathbf{d} \mathrm{WSe}_{2}$ on the freestanding graphene membrane [78]. Copyright 2015, American Chemical Society. e SEM image of WS $_{2}$ on h-BN flake [80]. Copyright 2014, American Chemical Society

monolayer growth [78]. Although $\mathrm{MoS}_{2}$ grains showed random orientations on graphene according to previous results, at the same time, other work showed that sides of single-crystalline $\mathrm{MoS}_{2}$ preferred parallel orientation at one sides of underlying graphene, and the SAED result also confirmed the lattice orientations of $\mathrm{MoS}_{2}$ and graphene coincided [79]. Similarly, another group observed distinct crystallographic orientation of $\mathrm{WS}_{2}$ on h-BN with two different orientations with $60^{\circ}$ angle, shown in Fig. 4e. Unlike graphene, the difference in electronegativity between $\mathrm{B}$ and $\mathrm{N}$ atoms results coulombic interaction played a vital role for the limited crystallographic orientation of $\mathrm{WS}_{2}$ on h-BN substrate [80].

\subsection{Morphology}

The morphology control is also important for scalable synthesis of high quality TMDs, which further ensures high quality and high performance for applications in optoelectronic and electronic properties. Single-layered TMDs with different morphologies, like triangles, stars, pentagons, hexagons, etc. have been synthesized by CVD methods [81]. Many researchers demonstrated that the morphologies of TMDs layers are dependent on the growth conditions, including substrates, gas flow rate, temperature, precursors ratio and concentration, etc. For example, it is found that the hydrogen has substantial effect on the morphologies of TMDs during growth, and the shape of monolayer triangle $\mathrm{WS}_{2}$ can be tailored from jagged to straight edge by adding minor amount $\mathrm{H}_{2}$ gas [82]. Similarly, with increasing $\mathrm{H}_{2}$ concentration, more $\mathrm{WO}_{3}$ were reduced by $\mathrm{H}_{2}$ to form volatile $\mathrm{WO}_{3-\mathrm{x}}$, by depositing $\mathrm{W}$ atoms onto Se edges. While appropriate fluxes of $\mathrm{WO}_{3-\mathrm{x}}$ and $\mathrm{Se}$ enables the formation of triangles with $\mathrm{W} / \mathrm{Se}$ edges or hexagon with high selectivity by controlling the $\mathrm{H}_{2}$ pressure [83]. Moreover, the distance between metal source and substrate, which determines the concentration of vapor metal precursor, plays a crucial role in the $\mathrm{MoS}_{2}$ morphology control [84]. With the increasing of distance from $\mathrm{MoO}_{3}$ precursor, $\mathrm{MoS}_{2}$ is transformed from mid-sized truncated triangle of $\sim 6 \mu \mathrm{m}$ to large triangle of $\sim 50 \mu \mathrm{m}$, then increase to medium-sized truncated triangle, followed by small hexagon, and finally tiny triangle of $\sim 2-3 \mu \mathrm{m}$ (Fig. 5a). This shape evolution parameters was found to strongly depends on the growth rates of different edge terminations. When 


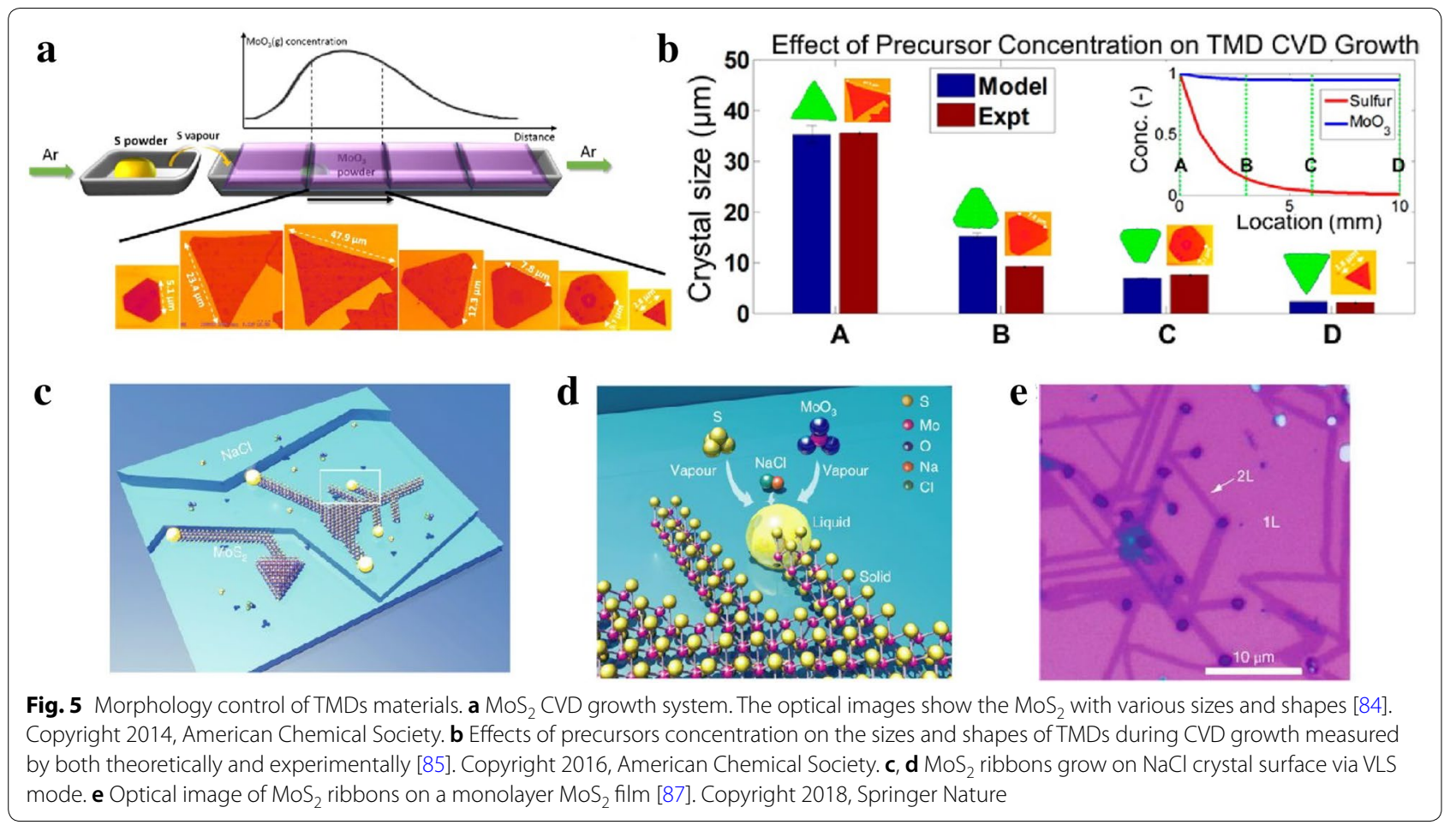

the Mo: $\mathrm{S}$ ratio was equal to $1: 2$, the growth rate of different edge terminations was equal, which resulted hexagon shape. On the other hand, when Mo: S ratio was larger than 1:2, the S-zigzag (zz) terminations grew faster than the Mo-zz terminations because the exposed $\mathrm{S}$ atoms had higher probability to bond with free Mo atoms, thus the final structure turned to triangles with Mo-zz termination sides. Conversely, when the substrate was far away from Mo source, the concentration of Mo vapor was decreased while the $S$ vapor gradient was small, then the Mo: $\mathrm{S}$ ratio was smaller than 1:2, and S-zz triangles were obtained. Meanwhile, some work proposed that the Mo-zz triangle has sharp and straight edges than S-zz triangle, which allowed the identification of crystal orientation by optical microscopy [68]. Furthermore, a growth model is developed using KMC simulations to predict the $\mathrm{MoS}_{2}$ variation in shape and size as a function of precursor concentration (Fig. 5b) [85]. In addition, a combined experimental and numerical simulation work is used to control the synthesis of vertical to planar $2 \mathrm{D} \mathrm{MoS}_{2}$. According to the result, the transition from vertical to planar triangle and thin film growth was achieved by placing substrate at different position with different orientations and changing the carrier gas flow rate. When the substrate was normal to the flow stream, the vertically standing $\mathrm{MoS}_{2}$ nanosheets were the dominant structures; while planar triangle and thin film were synthesized by placing the substrate facing down on top of the crucible and facing up next to metal source. The concentration gradient and distribution of the precursor play the critical role to control the growth mode and density, resulting in different orientations and morphologies [86]. Very recently, a vapor-liquid-solid (VLS) mode has been promoted to grow monolayer $\mathrm{MoS}_{2}$ nanoribbons. The liquid phase $\mathrm{Na}-\mathrm{Mo}-\mathrm{O}$ droplet formed by alkali metal halide reacting with metal oxide precursors that worked as intermediate and crawl on the substrate (Fig. 5c, d) [87]. Using this method, locally well-defined $\mathrm{MoS}_{2}$ ribbons have been further grown on a continuous $\mathrm{MoS}_{2}$ monolayer film, which pre-grown on a $\mathrm{SiO}_{2} /$ $\mathrm{Si}$ substrate (Fig. 5e). To meet the practical demands, lots of attempts have been made to synthesize largescale continuous 2D binary films or lateral heterostructures for electronics, optoelectronics, sensors, or other flexible devices. For example, a two-step CVD method is reported for epitaxial growth of lateral $\mathrm{WSe}_{2}-\mathrm{MoS}_{2}$ heterojunction with an atomically sharp interface. This kinds of TMDs lateral heterojunctions are key components for $\mathrm{p}-\mathrm{n}$ rectifying diodes, light-emitting diodes (LED), photodetectors, and photovoltaic devices, etc. [88]. Recently, $2 \mathrm{H}-\mathrm{MoTe}_{2} / \mathrm{MoS}_{2}$ were synthesized by using a magnet-assisted precursor delivery system. Based on this heterostructure, the vertical $\mathrm{p}-\mathrm{n}$ junction shows a broadband photo-response range from UV $(200 \mathrm{~nm})$ to near-infrared (1100 nm) (Fig. 6a) [89]. 

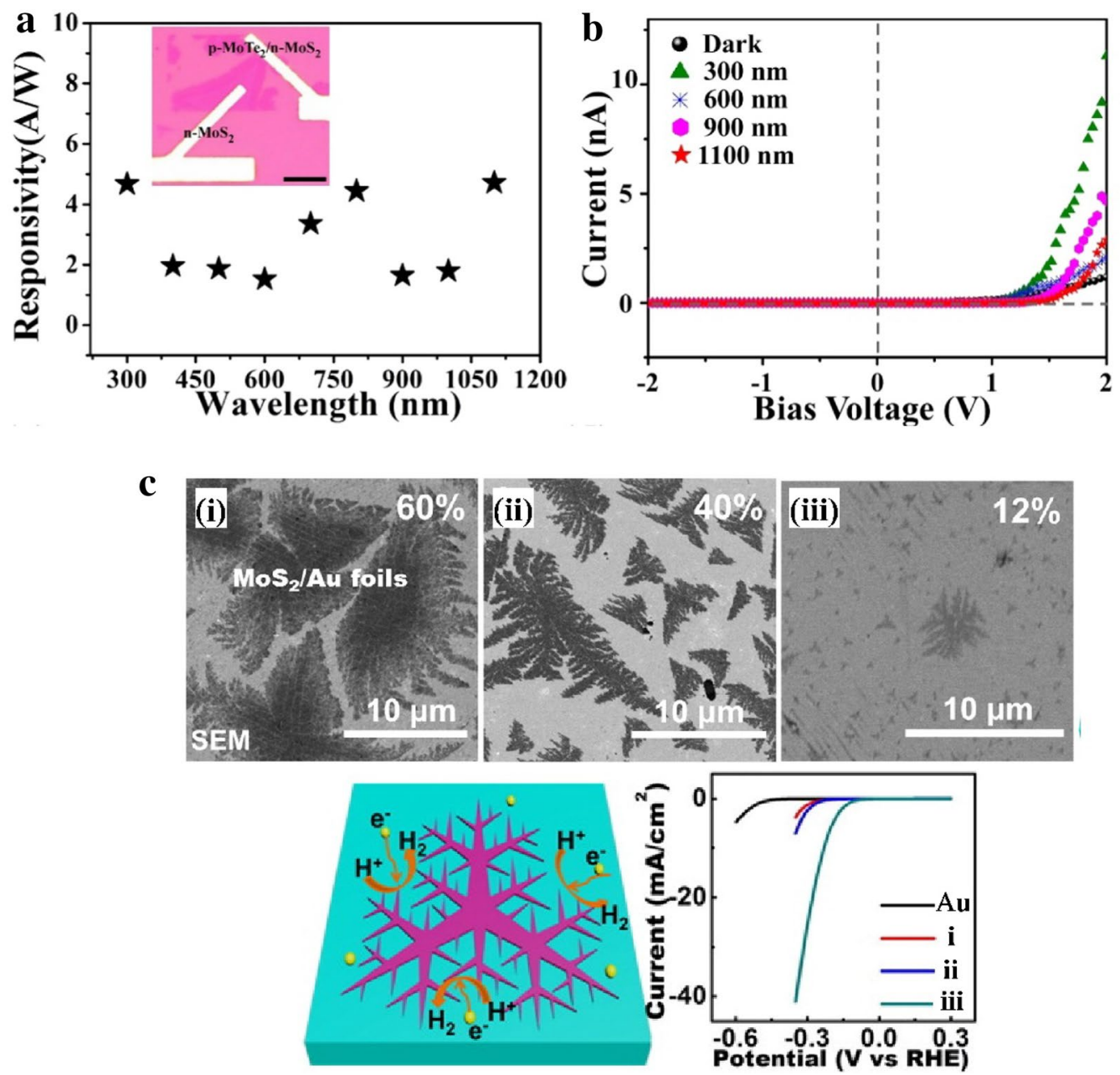

Fig. 6 Optoelectronic properties and electrochemical catalysis applications of TMDs materials. a Spectral responsivity curve of the $\mathrm{MoTe}_{2} / \mathrm{MoS}_{2}$ heterostructures $\mathbf{b} \mid-V$ curves of the phototransistor device [89]. Copyright 2018, Elsevier B.V. c SEM images of different coverages of dendritic MoS 2 and their polarization curves [95]. Copyright 2014, American Chemical Society

Figure $6 \mathrm{~b}$ illustrates the $\mathrm{MoTe}_{2} / \mathrm{MoS}_{2}$ device shows a photovoltaic $\mathrm{p}-\mathrm{n}$ junction effect under bias voltage for incidental lights, compared with dark environment. Other work also demonstrated that $\mathrm{MoS}_{2}-\mathrm{MoS}_{2} /$ CNT heterostructure exhibits attractive electrical and mechanical properties for flexible optoelectronics [90]. However, for electrochemical catalysis application and energy storage, like hydrogen evolution reaction (HER) and battery materials, it has been proven that the active sites are mainly at the edges or defects of $\mathrm{MoS}_{2}$. The exchange current density shows a linear dependence on the $\mathrm{MoS}_{2}$ edge, rather than area coverage $[91,92]$. Only the surface of few TMDs martials from group $\mathrm{V}$ (such as $\mathrm{V}, \mathrm{Nb}$, and $\mathrm{Ta}$ ) showed activity for electrolysis of water [93]. Besides these, sufficient amounts of efforts have been drawn to grow vertically aligned or dendritic TMDs to increase active site for
HER. For example, synthesized $\mathrm{MoS}_{2}$ and $\mathrm{MoSe}_{2}$ film with vertically aligned layers are compared and confirmed the exchange current density has direct correlation with the density of the exposed edge sites [28]. Similarly, horizontally and vertically aligned $\mathrm{MoS}_{2}$ by CVD method has shown that the vertically aligned $\mathrm{MoS}_{2}$ with a hydrophobic surface exhibit better HER performance [94]. On the other hand, much attention has been paid on the substrate effect for synthesizing dendritic $\mathrm{MoS}_{2}$ on specific substrates. Dendritic, monolayer $\mathrm{MoS}_{2}$ flakes on $\mathrm{SrTiO}_{3}$ (STO) is obtained and the tunable degrees of fractal shape provide abundant edges and serve as effective electrocatalysts for HER (Fig. 6c) [95]. After that, it is also reported that dendritic $\mathrm{MoS}_{2}$ on $\mathrm{LaAlO}_{3}(100)$ is also engineered to expose more active edge sites with high nucleation density [96]. Our group has also demonstrate the growth of single-crystal 
$\mathrm{MoSe}_{2}$ on $\mathrm{N}$-doped graphene where polymer worked as an absorption matrix to mitigate aggregation, and the $\mathrm{H}_{2}$ etching step during CVD treatment provided extra edge sites for $\mathrm{H}_{2}$ evolution in the inert basal plane [97].

Recently, the evolution of novel structures of TMCs, like polygonal twins, screw dislocations, etc. is also widely studied because the possibility of new functions. The merging of single-crystal or changes of layer stacking not only form different morphologies, but also affect the optical, electronic, and magnetic properties of TMCs. Recent report has shown that the preparation of a spirals of layered $\mathrm{MoS}_{2}$ with AA lattice stacking morphology (contrast to centrosymmetric $\mathrm{AB}$ stacking in intrinsic $\mathrm{MoS}_{2}$ ), this non-centrosymmetric spiral leads to secondharmonic generation (SHG) signal [98]. It is further confirmed that the triangular spiral $\mathrm{WSe}_{2}$ plates with strong SHG and enhanced PL signal, and the plates contain both triangular and hexagonal dislocations with diverse mixed properties [99]. Wulff constructions is used for bowtie- and star-shape $\mathrm{MoS}_{2}$ twin-islands (high-symmetry poly-crystals with $60^{\circ}$ lattice misorientation angles) under thermodynamic and or kinetic control conditions. Based on the comparison of the length of grain boundary and base edge, it is possible to distinguish the thermodynamic and kinetic control by measuring the aspect ratio of bowtie shape. The phase field model was also employed to simulate the growth of $\mathrm{MoS}_{2}$ and symmetric shape evolution [72]. Successfully growth of dendrites $\mathrm{MoS}_{2}$ is demonstrated by pretreating substrates with adhesive tapes and controlling S:Mo vapor ratio. The successive nucleation of twin crystals at the side edges contributed to the sub-branch growth, and the accumulated sulfur vacancies in cyclic twin regions lead to strong and localized enhancement of PL emission, determined the shape dependency of optical property [100].

\section{Summary and outlook}

Recent progress of 2D TMDs materials brings lots of advances and opportunities in the scientific community with numerous concepts and technologies. There have been many breakthroughs in the synthesis of TMDs layers by CVD techniques in the past several years. In this review, we analyze the possibility of controllable growth of 2D TMDs by CVD method based on the discussion of growth routes and mechanisms. The control of grain size, boundaries, orientation and morphology are highlighted, aiming to bring inspiration to future research in this field.

Although the latest published researches have successfully achieved the growth of high quality inch-size TMDs, it is still challenging to realize the controllable growth of wide range of TMDs with desired layer number, large domain size, target orientation and morphologies. One of the most important reason is that most of the precursors of TMDs are solid-state in common CVD growth system, except MOCVD. Unlike gas sources for graphene, it is difficult to control and maintain the concentrations of precursors precisely over whole growth process. Additionally, by using inductively coupled plasma enhanced CVD, graphene can be synthesized at temperature as low as $300{ }^{\circ} \mathrm{C}$, but it is still challenging to grow TMDs at such low temperature. These show the importance of development of new CVD system, such as using external heating equipment, or introducing additives to lower melting points and reaction barriers. Meanwhile, suitable analytical model combined with simulations and calculations should be further developed to reveal the growth mechanism and set reliable theoretical predictions to control the growth experimentally.

On the other hand, 2D TMDs layers are promising materials for different kinds of electronics and optoelectronics devices. Although most of the TMDs based on VIB group metal, like $\mathrm{MoS}_{2}, \mathrm{WS}_{2}, \mathrm{MoSe}_{2}$ and $\mathrm{WSe}_{2}$ have widely been synthesized by CVD method, the growth of other novel layered materials may open up the possibility to explore the applications of 2D materials. Furthermore, the defects engineering, grain boundaries and phase transitions of TMDs display tremendous opportunity for specific area of applications. Additionally, we mainly focused on the synthesis of binary compounds in this review, but it is worth noting that the alloying of semiconductors and construction of heterostructures are ideal ways to tailoring the band structure of TMDs. Hence, the design and preparation of novel TMDs heterostructures as well as alloys is also important aspects to create new practical applications. Some other issues, such as the direct growth on flexible substrates or a successful method to transfer the TMDs on the target substrate after growth is necessary for the fabrication of most electronic devices. Therefore, considering the growth and application of 2D TMDs layers are still at the exploration stage, there are still numerous possibilities and strong demand to further understanding and developing the synthesis of 2D TMDs materials.

\section{Authors' contributions}

JWY, MDH and ZTL contributed to the preparation of the manuscript. All authors read and approved the final manuscript.

\section{Authors' information}

Miss Jiawen You received her B.S. degree in Applied Chemistry from Donghua University in 2016. Now she is pursuing her MPhil. degree under the supervision of Prof. Zhengtang Luo at Department of Chemical and Biological

Engineering (CBE), Hong Kong University of Science and Technology (HKUST). Her current research interests mainly focus on the CVD growth of 2D materials and their heterostructures for optoelectronics.

Mr. Md Delowar Hossain received his B.Sc. and M.Sc. degree in Applied Chemistry and Chemical Engineering from University of Rajshahi, Bangladesh. Now he is pursuing his Ph.D. degree under the supervision of Prof. Tom Luo at CBE, HKUST. His research interests are the theoritical and experimental 
investigation to find the origin of catalysts activity in case of 2D material applied for different energy applications.

Prof. Zhengtang Luo is an Associate Professor in Hong Kong University of Science and Technology (HKUST). He earned his B.S. (1998) and M.S. (2001) degrees from South China University of Technology (SCUT), and a Ph.D. degree from University of Connecticut (2007). After working as a postdoctoral researcher at University of Pennsylvania (UPenn), he joined CBE, HKUST at 2012. His research mainly focuses on materials chemistry, materials physics and the nanofabrication of semiconductor nano-bioelectronics.

\section{Acknowledgements}

Technical assistance from the Materials Characterization and Preparation Facilities is greatly appreciated.

\section{Competing interests}

The authors declare that they have no competing interests.

\section{Availability of data and materials}

Not applicable.

\section{Funding}

This project was supported by the Innovation and Technology Commission (ITC-CNERC14SC01), the Research Grant Council of Hong Kong SAR (Project Number 16204815), NSFC-RGC Joint Research Scheme (N_HKUST607/17).

\section{Publisher's Note}

Springer Nature remains neutral with regard to jurisdictional claims in published maps and institutional affiliations.

Received: 2 August 2018 Accepted: 10 September 2018 Published online: 28 September 2018

\section{References}

1. M. Chhowalla, H.S. Shin, G. Eda, L.-J. Li, K.P. Loh, H. Zhang, Nat. Chem. 5, 263 (2013)

2. T. Kanazawa, T. Amemiya, A. Ishikawa, V. Upadhyaya, K. Tsuruta, T. Tanaka, Y. Miyamoto, Sci. Rep. 6, 22277 (2016)

3. R. Ganatra, Q. Zhang, ACS Nano 8, 4074-4099 (2014)

4. C. Chunxiao, S. Jingzhi, W. Yanlong, Y. Ting, Adv. Opt. Mater. 6, 1700767 (2018)

5. J.C.E. Rasch, T. Stemmler, B. Müller, L. Dudy, R. Manzke, Phys. Rev. Lett. 101,237602 (2008)

6. P. Li, Y. Wen, X. He, Q. Zhang, C. Xia, Z.-M. Yu, S.A. Yang, Z. Zhu, H.N. Alshareef, X.X. Zhang, Nat. Commun. 8, 2150 (2017)

7. C. Wang, X. Wu, Y. Ma, G. Mu, Y. Li, C. Luo, H. Xu, Y. Zhang, J. Yang, X. Tang, J. Zhang, W. Bao, C. Duan, J. Mater. Chem. A 6, 8299-8306 (2018)

8. Z. Sihan, H. Takato, K. Takashi, W. Kenji, T. Takashi, S. Katsuaki, T. Takashi, S. Hisanori, K. Ryo, 2D Mater. 3, 025027 (2016)

9. R.A. Gordon, D. Yang, E.D. Crozier, D.T. Jiang, R.F. Frindt, Phys. Rev. B 65, 125407 (2002)

10. A. Kuc, N. Zibouche, T. Heine, Phys. Rev. B 83, 245213 (2011)

11. B. Sipos, A.F. Kusmartseva, A. Akrap, H. Berger, L. Forró, E. Tutiš, Nat. Mater. 7, 960 (2008)

12. A. Splendiani, L. Sun, Y. Zhang, T. Li, J. Kim, C.Y. Chim, G. Galli, F. Wang, Nano Lett. 10, 1271-1275 (2010)

13. X. Xi, L. Zhao, Z. Wang, H. Berger, L. Forró, J. Shan, K.F. Mak, Nat. Nanotechnol. 10, 765 (2015)

14. B. Radisavljevic, A. Radenovic, J. Brivio, V. Giacometti, A. Kis, Nat. Nanotechnol. 6, 147 (2011)

15. H. Wang, L. Yu, Y.-H. Lee, Y. Shi, A. Hsu, M.L. Chin, L.J. Li, M. Dubey, J. Kong, T. Palacios, Nano Lett. 12, 4674-4680 (2012)

16. Y. Yoon, K. Ganapathi, S. Salahuddin, Nano Lett. 11, 3768-3773 (2011)

17. Z. Yin, H. Li, H. Li, L. Jiang, Y. Shi, Y. Sun, G. Lu, Q. Zhang, X. Chen, H. Zhang, ACS Nano 6, 74-80 (2012)

18. G.L. Frey, K.J. Reynolds, R.H. Friend, H. Cohen, Y. Feldman, J. Am. Chem. Soc. 125, 5998-6007 (2003)
19. H.S. Lee, S.-W. Min, Y.-G. Chang, M.K. Park, T. Nam, H. Kim, J.H. Kim, S. Ryu, S. Im, Nano Lett. 12, 3695-3700 (2012)

20. C. Zhong, C. Duan, F. Huang, H. Wu, Y. Cao, Chem. Mater. 23, 326-340 (2011)

21. K.S. Novoselov, D. Jiang, F. Schedin, T.J. Booth, V.V. Khotkevich, S.V. Morozov, A.K. Geim, Proc. Natl. Acad. Sci. USA 102, 10451-10453 (2005)

22. C. Lee, Q. Li, W. Kalb, X.-Z. Liu, H. Berger, R.W. Carpick, J. Hone, Science 328, 76-80 (2010)

23. D. Golberg, Nat. Nanotechnol. 6, 200 (2011)

24. J.N. Coleman, M. Lotya, A. O'Neill, S.D. Bergin, P.J. King, U. Khan, K. Young, A. Gaucher, S. De, R.J. Smith, I.V. Shvets, S.K. Arora, G. Stanton, H..Y. Kim, K. Lee, G.T. Kim, G.S. Duesberg, T. Hallam, J.J. Boland, J.J. Wang, J.F. Donegan, J.C. Grunlan, G. Moriarty, A. Shmeliov, R.J. Nicholls, J.M. Perkins, E.M. Grieveson, K. Theuwissen, D.W. McComb, P.D. Nellist, V. Nicolosi, Science 331, 568-571 (2011)

25. G. Eda, H. Yamaguchi, D. Voiry, T. Fujita, M. Chen, M. Chhowalla, Nano Lett. 11, 5111-5116 (2011)

26. L. Yi-Hsien, Z. Xin-Quan, Z. Wenjing, C. Mu-Tung, L. Cheng-Te, C. Kai-Di, Y. Ya-Chu, W.J. Tse-Wei, C. Chia-Seng, L. Lain-Jong, L. Tsung-Wu, Adv. Mater. 24, 2320-2325 (2012)

27. Y.-C. Lin, W. Zhang, J.-K. Huang, K.-K. Liu, Y.-H. Lee, C.-T. Liang, C.-W. Chu, L.-J. Li, Nanoscale 4, 6637-6641 (2012)

28. D. Kong, H. Wang, J.J. Cha, M. Pasta, K.J. Koski, J. Yao, Y. Cui, Nano Lett. 13 1341-1347 (2013)

29. Z. Yongjie, L. Zheng, N. Sina, P.M. Ajayan, L. Jun, Small 8, 966-971 (2012)

30. J.-T. Jang, S. Jeong, J.-W. Seo, M.-C. Kim, E. Sim, Y. Oh, S. Nam, B. Park, J. Cheon, J. Am. Chem. Soc. 133, 7636-7639 (2011)

31. S. Jeong, D. Yoo, J.-T. Jang, M. Kim, J. Cheon, J. Am. Chem. Soc. 134, 18233-18236 (2012)

32. H. Zhang, ACS Nano 9, 9451-9469 (2015)

33. Z. Zhiyuan, S. Ting, Z. Jixin, H. Xiao, Y. Zongyou, L. Gang, F. Zhanxi, Y. Qingyu, H.H. Hoon, Z. Hua, Angew. Chem. Int. Ed. 51, 9052-9056 (2012)

34. Y. Zhang, L. Zhang, C. Zhou, Acc. Chem. Res. 46, 2329-2339 (2013)

35. A. Reina, X. Jia, J. Ho, D. Nezich, H. Son, V. Bulovic, M.S. Dresselhaus, J. Kong, Nano Lett. 9, 30-35 (2009)

36. Q. Ji, Y. Zhang, Y. Zhang, Z. Liu, Chem. Soc. Rev. 44, 2587-2602 (2015)

37. Y. Shi, H. Li, L.J. Li, Chem. Soc. Rev. 44, 2744-2756 (2015)

38. J.L. Brito, M. Ilija, P. Hernández, Thermochim. Acta 256, 325-338 (1995)

39. K.-K. Liu, W. Zhang, Y.-H. Lee, Y.-C. Lin, M.-T. Chang, C.-Y. Su, C.-S. Chang, H. Li, Y. Shi, H. Zhang, C.-S. Lai, L.-J. Li, Nano Lett. 12, 1538-1544 (2012)

40. R. Ionescu, B. Campbell, R. Wu, E. Aytan, A. Patalano, I. Ruiz, S.W. Howell, A.E. McDonald, T.E. Beechem, K.A. Mkhoyan, M. Ozkan, C.S. Ozkan, Sci. Rep. 7, 6419 (2017)

41. Y. Lei, S. Pakhira, K. Fujisawa, X. Wang, O.O. Iyiola, N. Perea López, A. Laura Elías, L. Pulickal Rajukumar, C. Zhou, B. Kabius, N. Alem, M. Endo, R. Lv, J.L. Mendoza-Cortes, M. Terrones, ACS Nano 11, 5103-5112 (2017)

42. G.H. Han, N.J. Kybert, C.H. Naylor, B.S. Lee, J. Ping, J.H. Park, J. Kang, S.Y. Lee, Y.H. Lee, R. Agarwal, A.T.C. Johnson, Nat. Commun. 6, 6128 (2015)

43. J.-G. Song, J. Park, W. Lee, T. Choi, H. Jung, C.W. Lee, S.-H. Hwang, J.M. Myoung, J.-H. Jung, S.-H. Kim, C. Lansalot-Matras, H. Kim, ACS Nano 7, 11333-11340 (2013)

44. X. Shengyong, Y. Yong, X. Sishen, P. Lianmao, NanoMicro Lett. 2, 184-189(2010)

45. X. Wang, H. Feng, Y. Wu, L. Jiao, J. Am. Chem. Soc. 135, 5304-5307 (2013)

46. C. Ying, G. Lin, L. Huiqiao, M. Ying, Z. Tianyou, Adv. Mater. 29, 1603550 (2017)

47. S. Wu, C. Huang, G. Aivazian, J.S. Ross, D.H. Cobden, X. Xu, ACS Nano 7 , 2768-2772 (2013)

48. J.C. Shaw, H. Zhou, Y. Chen, N.O. Weiss, Y. Liu, Y. Huang, X. Duan, Nano Res. 7, 511-517 (2014)

49. C. Jung, S.M. Kim, H. Moon, G. Han, J. Kwon, Y.K. Hong, I. Omkaram, Y. Yoon, S. Kim, J. Park, Sci. Rep. 5, 15313 (2015)

50. J.-K. Huang, J. Pu, C.-L. Hsu, M.-H. Chiu, Z.-Y. Juang, Y.-H. Chang, W.-H. Chang, Y. Iwasa, T. Takenobu, L.J. Li, ACS Nano 8, 923-930 (2014)

51. Y. Dan, H. Xiaozong, Z. Minghao, D. Yao, Z. Shasha, L. Aoju, Y. Yiwei, L. Huiqiao, L. Zhengtang, G. Lin, Z. Tianyou, Adv. Funct. Mater. 28, 1800785 (2014)

52. J. Zhou, J. Lin, X. Huang, Y. Zhou, Y. Chen, J. Xia, H. Wang, Y. Xie, H. Yu, J. Lei, D. Wu, F. Liu, Q. Fu, Q. Zeng, C.-H. Hsu, C. Yang, L. Lu, T. Yu, Z. Shen, H. Lin, B.I. Yakobson, Q. Liu, K. Suenaga, G. Liu, Z. Liu, Nature 556, 355-359 (2018) 
53. X. Ling, Y.-H. Lee, Y. Lin, W. Fang, L. Yu, M.S. Dresselhaus, J. Kong, Nano Lett. 14, 464-472 (2014)

54. W.Y. Lee, T.M. Besmann, M.W. Stott, J. Mater. Res. 9, 1474-1483 (1994)

55. Y. Yu, C. Li, Y. Liu, L. Su, Y. Zhang, L. Cao, Scientific Reports 2013, 3 (1866)

56. S.-L. Shang, G. Lindwall, Y. Wang, J.M. Redwing, T. Anderson, Z.-K. Liu, Nano Lett. 16, 5742-5750 (2016)

57. H. Ye, J. Zhou, D. Er, C.C. Price, Z. Yu, Y. Liu, J. Lowengrub, J. Lou, Z. Liu, V.B. Shenoy, ACS Nano 11, 12780-12788 (2017)

58. S. Xing, W. Wu, Y. Wang, J. Bao, S.-S. Pei, Chem. Phys. Lett. 580, 62-66 (2013)

59. Y. Nie, C. Liang, P.-R. Cha, L. Colombo, R.M. Wallace, K. Cho, Sci. Rep. 7 $2977(2017)$

60. J. Zhang, H. Yu, W. Chen, X. Tian, D. Liu, M. Cheng, G. Xie, W. Yang, R. Yang, X. Bai, D. Shi, G. Zhang, ACS Nano 8, 6024-6030 (2014)

61. Y. Milad, W. Xufei, G. Tushar, G. Debjit, X. Lixin, Z. Zhuan, B. Hatem, B. Jiming, C. Shuo, L. Tengfei, K. Nikhil, M. Anastassios, Adv. Funct. Mater. 27, 1704357 (2017)

62. Z. Lin, Y. Zhao, C. Zhou, R. Zhong, X. Wang, Y.H. Tsang, Y. Chai, Sci. Rep. 5 , $18596(2015)$

63. Y.-F. Lim, K. Priyadarshi, F. Bussolotti, P.K. Gogoi, X. Cui, M. Yang, J. Pan, S.W. Tong, S. Wang, S.J. Pennycook, K.E.J. Goh, A.T.S. Wee, S.L. Wong, D. Chi, ACS Nano 12, 1339-1349 (2018)

64. J. Chen, X. Zhao, S.J.R. Tan, H. Xu, B. Wu, B. Liu, D. Fu, W. Fu, D. Geng, Y. Liu, W. Liu, W. Tang, L. Li, W. Zhou, T.C. Sum, K.P. Loh, J. Am. Chem. Soc. 139 1073-1076 (2017)

65. P. Yang, X. Zou, Z. Zhang, M. Hong, J. Shi, S. Chen, J. Shu, L. Zhao, S. Jiang, X. Zhou, Y. Huan, C. Xie, P. Gao, Q. Chen, Q. Zhang, Z. Liu, Y. Zhang, Nat. Commun. 9, 979 (2018)

66. J. Shi, X. Zhang, D. Ma, J. Zhu, Y. Zhang, Z. Guo, Y. Yao, Q. Ji, X. Song, Y. Zhang, C. Li, Z. Liu, W. Zhu, Y. Zhang, ACS Nano 9, 4017-4025 (2015)

67. S. Najmaei, Z. Liu, W. Zhou, X. Zou, G. Shi, S. Lei, B.I. Yakobson, J.-C. Idrobo, P.M. Ajayan, J. Lou, Nat. Mater. 12, 754 (2013)

68. A.M. van der Zande, P.Y. Huang, D.A. Chenet, T.C. Berkelbach, Y. You, G.-H Lee, T.F. Heinz, D.R. Reichman, D.A. Muller, J.C. Hone, Nat. Mater. 12, 554 (2013)

69. Z. Liu, M. Amani, S. Najmaei, Q. Xu, X. Zou, W. Zhou, T. Yu, C. Qiu, A.G. Birdwell, F.J. Crowne, R. Vajtai, B.I. Yakobson, Z. Xia, M. Dubey, P.M. Ajayan, J. Lou, Nat. Commun. 5, 5246 (2014)

70. W. Zhou, X. Zou, S. Najmaei, Z. Liu, Y. Shi, J. Kong, J. Lou, P.M. Ajayan, B.I. Yakobson, J-C. Idrobo, Nano Lett. 13, 2615-2622 (2013)

71. Y.L. Huang, Y. Chen, W. Zhang, S.Y. Quek, C.-H. Chen, L.-J. Li, W.-T. Hsu, W.-H. Chang, Y.J. Zheng, W. Chen, A.T.S. Wee, Nat. Commun. 6, 6298 (2015)

72. V.I. Artyukhov, Z. Hu, Z. Zhang, B.I. Yakobson, Nano Lett. 16, 3696-3702 (2016)

73. D. Ruzmetov, K. Zhang, G. Stan, B. Kalanyan, G.R. Bhimanapati, S.M. Eichfeld, R.A. Burke, P.B. Shah, T.P. O'Regan, F.J. Crowne, A.G. Birdwell, J.A. Robinson, A.V. Davydov, T.G. Ivanov, ACS Nano 10, 3580-3588 (2016)

74. L. Chen, B. Liu, M. Ge, Y. Ma, A.N. Abbas, C. Zhou, ACS Nano 9, 8368$8375(2015)$

75. A. Aljarb, Z. Cao, H.-L. Tang, J.-K. Huang, M. Li, W. Hu, L. Cavallo, L.-J. Li, ACS Nano 11, 9215-9222 (2017)

76. Q. Ji, Y. Zhang, T. Gao, Y. Zhang, D. Ma, M. Liu, Y. Chen, X. Qiao, P.-H. Tan, M. Kan, J. Feng, Q. Sun, Z. Liu, Nano Lett. 13, 3870-3877 (2013)
77. H.G. Ji, Y.-C. Lin, K. Nagashio, M. Maruyama, P. Solís-Fernández, A. Sukma Aji, V. Panchal, S. Okada, K. Suenaga, H. Ago, Chem. Mater. 30, 403-411 (2018)

78. A. Azizi, S. Eichfeld, G. Geschwind, K. Zhang, B. Jiang, D. Mukherjee, L. Hossain, A.F. Piasecki, B. Kabius, J.A. Robinson, N. Alem, ACS Nano 9, 4882-4890 (2015)

79. H. Ago, H. Endo, P. Solís-Fernández, R. Takizawa, Y. Ohta, Y. Fujita, K. Yamamoto, M. Tsuji, ACS Appl. Mater. Interfaces 7, 5265-5273 (2015)

80. M. Okada, T. Sawazaki, K. Watanabe, T. Taniguch, H. Hibino, H. Shinohara, R. Kitaura, ACS Nano 8, 8273-8277 (2014)

81. G. Zhang, J. Wang, Z. Wu, R. Shi, W. Ouyang, A. Amini, B.N. Chandrashekar, N. Wang, C. Cheng, ACS Appl. Mater. Interfaces 9, 763-770 (2017)

82. Y. Zhang, Y. Zhang, Q. Ji, J. Ju, H. Yuan, J. Shi, T. Gao, D. Ma, M. Liu, Y. Chen, X. Song, H.Y. Hwang, Y. Cui, Z. Liu, ACS Nano 7, 8963-8971 (2013)

83. C. Jianyi, L. Bo, L. Yanpeng, T. Wei, N.C. Tai, L. Linjun, Z. Jian, G. Libo, Z. Yi, S.H. Suk, J.H. Young, L.K. Ping, Adv. Mater. 27, 6722-6727 (2015)

84. S. Wang, Y. Rong, Y. Fan, M. Pacios, H. Bhaskaran, K. He, J.H. Warner, Chem. Mater. 26, 6371-6379 (2014)

85. A. Govind Rajan, J.H. Warner, D. Blankschtein, M.S. Strano, ACS Nano 10 4330-4344 (2016)

86. Z. Fu, M. Kasra, A. Mohammed Abu, A. Amin, F.H. Mel Jr., M.R. Joan, C. Long-Qing, A. Nasim, 2D Mater. 4, 025029 (2017)

87. S. Li, Y.-C. Lin, W. Zhao, J. Wu, Z. Wang, Z. Hu, Y. Shen, D.-M. Tang, J. Wang, Q. Zhang, H. Zhu, L. Chu, W. Zhao, C. Liu, Z. Sun, T. Taniguchi, M. Osada, W. Chen, Q.-H. Xu, A.T.S. Wee, K. Suenaga, F. Ding, G. Eda, Nat. Mater. 17, 535-542 (2018)

88. M.-Y. Li, Y. Shi, C.-C. Cheng, L.-S. Lu, Y.-C. Lin, H.-L. Tang, M.-L. Tsai, C.-W. Chu, K.-H. Wei, J.-H. He, W.-H. Chang, K. Suenaga, L.-J. Li, Science 349. 524-528 (2015)

89. Y. Ding, N. Zhou, L. Gan, X. Yan, R. Wu, I.H. Abidi, A. Waleed, J. Pan, X. Ou, Q. Zhang, M. Zhuang, P. Wang, X. Pan, Z. Fan, T. Zhai, Z. Luo, Nano Energy 49, 200-208 (2018)

90. L. Li, G. Yichuan, S. Yuping, Y. Long, Q. Liang, G. Shouliang, W. Jinfen, Q. Xiaohui, L. Hongbian, S. Yuanyuan, F. Ying, Adv. Mater. 30, 1706215 (2018)

91. T.F. Jaramillo, K.P. Jørgensen, J. Bonde, J.H. Nielsen, S. Horch, I. Chorkendorff, Science 317, 100-102 (2007)

92. G. Ye, Y. Gong, J. Lin, B. Li, Y. He, S.T. Pantelides, W. Zhou, R. Vajtai, P.M. Ajayan, Nano Lett. 16, 1097-1103 (2016)

93. H. Pan, Scientific Reports 4, 5348 (2014)

94. P. Kumar, B. Viswanath, CrystEngComm 19, 5068-5078 (2017)

95. Y. Zhang, Q. Ji, G.-F. Han, J. Ju, J. Shi, D. Ma, J. Sun, Y. Zhang, M. Li, X.-Y. Lang, Y. Zhang, Z. Liu, ACS Nano 8, 8617-8624 (2014)

96. B. Samaneh, M. Seyedeh Zahra, R. Ali, J. Phys. D Appl. Phys. 51, 195302 (2018)

97. M. Zhuang, Y. Ding, X. Ou, Z. Luo, Nanoscale 9, 4652-4659 (2017)

98. L. Zhang, K. Liu, A.B. Wong, J. Kim, X. Hong, C. Liu, T. Cao, S.G. Louie, F. Wang, P. Yang, Nano Lett. 14, 6418-6423 (2014)

99. M.J. Shearer, L. Samad, Y. Zhang, Y. Zhao, A. Puretzky, K.W. Eliceiri, J.C. Wright, R.J. Hamers, S. Jin, J. Am. Chem. Soc. 139, 3496-3504 (2017)

100. J. Wang, X. Cai, R. Shi, Z. Wu, W. Wang, G. Long, Y. Tang, N. Cai, W. Ouyang, P. Geng, B.N. Chandrashekar, A. Amini, N. Wang, C. Cheng, ACS Nano 12 635-643 (2018) 